\title{
Use and alternatives to coercive measures in inpatient residential care facilities for adults with disabilities: study protocol of a mixed-methods study
}

Malte Klemmt ( $\square$ malte.klemmt@fhws.de)

Peter Brieger

Thomas Schmitt-Schäfer

Annika Mörtz

Tanja Henking

\section{Short Report}

Keywords: coercive measures, physical restraint, chemical restraint, residential care facilities, adults with disabilities, survey, participating observation, focus groups, document analysis

Posted Date: January 13th, 2022

DOI: https://doi.org/10.21203/rs.3.rs-1257597/v1

License: (c) (1) This work is licensed under a Creative Commons Attribution 4.0 International License.

Read Full License 
Use and alternatives to coercive measures in inpatient residential care facilities for adults with disabilities: study protocol of a mixed-methods study

Malte Klemmt ${ }^{1}$, Peter Brieger ${ }^{2}$, Thomas Schmitt-Schäfer ${ }^{3}$, Annika Mörtz ${ }^{3}$, Tanja Henking ${ }^{1}$

${ }^{1}$ Institute of Applied Social Sciences, University of Applied Science Würzburg-Schweinfurt, Würzburg/Germany

${ }^{2}$ kbo-Isar-Amper-Klinikum, Medical Faculty, Ludwig-Maximilians-University Munich, Haar/Germany

${ }^{3}$ transfer - Enterprise for Social Innovations, Wittlich/Germany

Corresponding author:

Malte Klemmt

Institute of Applied Social Sciences, University of Applied Science Würzburg-Schweinfurt

Münzstraße 12, 97070 Würzburg/Germany

malte.klemmt@fhws.de 


\section{Abstract}

\section{Background}

In Germany, the use of coercive measures such as physical or chemical restraints in certain settings has been the focus of high court decisions, political debates, and scientific research in areas like psychiatric care. Such encroachments on fundamental rights could easily be overlooked in the case of residents in inpatient residential care facilities for adults with disabilities. However, little data are available on this issue. This study aims to investigate the types, frequencies, and characteristics of the use of coercive measures as well as the reasons and justifications of their use. Possible alternatives to these measures and contextual conditions will also be identified.

\section{Methods}

The study is based on an explorative, multi-phase, cross-sectional design, which consists of a mixedmethods approach including several forms of data collection and analysis bringing together forensic, sociological, pedagogical and medical perspectives and expertise. First, the entirety of the approximately $n=880$ residential care facilities for adults with disabilities in the study area will be surveyed by an online questionnaire. Participating passive observations will be carried out in $n=5$ selected residential care facilities ( 24 hours per facility), with an approximate total of $n=150$ residents. In these facilities, organizational documents such as house rules and residential documents such as medication plans will also be collected and analysed. Focus groups will be conducted in each of the five facilities including residents, staff, and legal guardians of the residents.

\section{Discussion}

This study will address important gaps in the current research by providing different forms of empirical data on the use or avoidance, causes, contexts, and alternatives to coercive measures in inpatient residential care facilities for adults with disabilities. This study is the first in Germany to combine a general overview (survey) and detailed insight (observations, document analysis, focus 
groups) approach to this issue. These findings will provide a basis for further research in the field as well as for the management of coercive measures in practical settings.

\section{Keywords}

coercive measures, physical restraint, chemical restraint, residential care facilities, adults with disabilities, survey, participating observation, focus groups, document analysis 


\section{Background}

The admissibility of coercive measures such as compulsory treatment, mechanical, physical, and chemical restraints or seclusion has received increasing attention in the past ten years due to several decisions by the Federal Constitutional Court [1] and the Federal Supreme Court in Germany. Most of the decisions in this area concerned patients subjected to coercive measures in involuntary psychiatric care. In Germany, coercive measures are permissible for patients in general psychiatric care who are considered incapable of giving consent and lack insight into their illness, based on the law of guardianship and the mental health acts [2]. In a recent decision on the use of restraints in psychiatric patients, the Federal Constitutional Court [3] emphasised the seriousness of the encroachment on fundamental rights; at the same time, restraint was deemed permissible in cases of danger to the self or others. In the field of psychiatry, a reduction in the use of restraints has been observed since this decision was handed down in 2018 [4]. This observation was made possible by the existence of systematically recorded data on the subject. The collection of data on the use of restraints in psychiatric patients was mandated by law in the aforementioned decision. The state of Baden-Württemberg, however, had already included this provision in its Mental Health Act of 2015 $[4,5]$. While awareness of the necessity of recording data on coercive measures in psychiatric institutions has increased, this has not been the case for residential care facilities (RCFs) for adults with disabilities. This study involves a comprehensive investigation of the use of coercive measures that restrict individual liberty through mechanical, psychical, and chemical restraint in the federal state of Bavaria/Germany which stands out in a comparison of federal states with over 880 RCFs. At the end of 2018, 83.400 adults with disabilities lived in RCFs in the state of Bavaria [6]. The people living in these RCFs do so due to mental and/or physical disabilities, sensory impairments, or substance use disorders [7]. In this area, the use of coercive measures such as restraints is permissible primarily based on the law on guardianship in cases of danger to self. Examples of these measures include physical and chemical restraint, seclusion, and the use of bed rails. In addition to the field of psychiatry [8-12], measures to reduce coercion and violence have also been discussed, 
and in some cases implemented, in the field of nursing homes [13-17]. However, little is known about the number of cases in which coercive measures are used, how they are justified, or the frequency and duration of restraint use in RCFs for adults with disabilities [18-20]. Due to the seriousness of the encroachment on fundamental rights associated with such coercive measures, the lack of knowledge about the actual use of these practices is unacceptable. Without more detailed knowledge about the types, frequencies, and reasons for coercive measures, there is no basis for a discussion about alternatives and the possibility of evaluating their effectiveness. The reliability of the information in this field, especially when collected from those responsible for the institutions themselves, has been identified as a problem. In addition, it is suspected that some coercive measures in this field are used under the guise of pedagogy [21], although from a legal standpoint, they appear to be coercive measures. A distinction must be made between measures that deprive people of their liberty and those that restrict it. Coercive measures can be defined are mechanical, physical, or chemical restraints that restrict freedom of movement against the will of the person concerned and have a fixed intensity and duration. These include, for example, seclusion, restraints such as bed rails and chair tables, as well as certain locking mechanisms. These methods should be distinguished from socalled informal coercion (through persuasion, threats, inducement) [22].

Awareness of the problems associated with obtaining meaningful data in these cases prompted us to adopt a mixed-methods approach that allows for the deepest possible insight into the setting and everyday life in the RCFs bringing together forensic, sociological, pedagogical and medical perspectives and expertise. The study aims to address the mentioned desideratum by generating empirically validated findings on the use or avoidance of coercive measures in RCFs for adults with disabilities in Bavaria. This goal also included an evaluation of individual motivations, reasons for justification, as well as personnel-related, organizational, and structural aspects of the facilities. The overall aim was to gain a comprehensive insight into everyday practices involving the use of restraints in the RCFs examined.

The study was guided by research questions related to three main themes: 
- Which types of coercive measures are used?

- How often are coercive measures used?

- For how long are coercive measures used?

- Are coercive measures used correctly and appropriately?

- For what types of disabilities or behavioural symptoms are coercive measures used most often?

Reasons for using coercive measures/restraints

- Which reasons are appropriate for the use of coercive measures?

- What is the justification for the use of coercive measures?

- How often do these reasons and justifications come into play?

Alternatives to coercive measures/restraints

- What alternatives could avoid coercive measures?

- Are there structural or personnel measures that could prevent the use of coercive measures?

- How does the use of alternatives to coercion affect the atmosphere in the RCFs, especially from the perspective of other residents and staff?

- Can best practices be developed for these situations?

The answers to these questions will provide fundamental knowledge about the actual practices used in these settings.

\section{Methods/Design}

\section{Setting and participants}

The study will take place in RCFs for adults with disabilities in the German state of Bavaria. Study participants include the heads of the facilities, staff, and the residents and their legal guardians. 
Residents who live in these RCFs have intellectual disabilities (45\%), mental disabilities (19\%), substance use disorders (6\%), physical disabilities (2\%), sensory impairments (2\%), or multiple disabilities (26\%) [7]. Actual living conditions were assumed to vary across participants.

\section{Study design}

The study has an explorative, multi-phase, cross-sectional design consisting of a mixed-methods approach that incorporates several methods of data collection and analysis allowing for the examination of different stakeholder perspectives. The study group is headed by a jurist (TH), a psychiatrist (PB) and social scientists (TSS, MK). The study consists of two parts, the first of which involves a survey of all RCFs in the study area, while the second entails the examination of five selected RCFs. This stage is further divided into three sub-parts. The study will involve the following procedures: an online survey of all inpatient RCFs for adults with disabilities within the investigation area (Part 1), followed by participating observations (Part 2a), analysis of resident records, medication use, and organizational documents such as working schedules (Part 2b), as well as focus groups with residents, their legal guardians and staff in five selected RCFs (Part 2c). The study phases take place in a predetermined sequence and are mutually dependent. For further details, see Fig. 1.

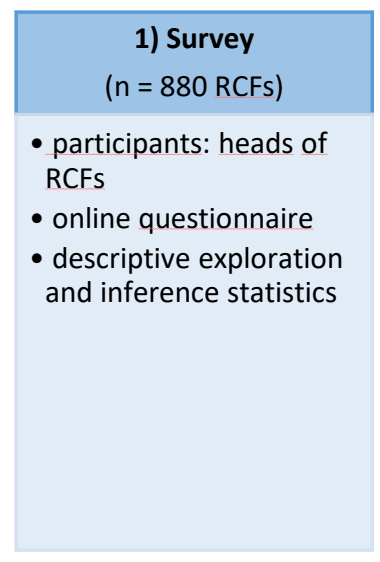

\begin{tabular}{l} 
2a) Observations \\
( $n=5$ RCFs) \\
- participants: residents \\
( $n=150$ ) and staff of \\
the RCFs \\
- passive, participating \\
observations \\
- structured protocolls \\
- descriptive exploration; \\
qualitative content \\
analysis (Gläser \& \\
Laudel) \\
\hline
\end{tabular}

2b) Document analysis
\[ (\mathrm{n}=5 \mathrm{RCFs} \text { ) } \]
- organizational
documents and
resident-documents ( $\mathrm{n}$
$=150$ )
- review of all
documents and
extraction on site
- descriptive exploration;
deductive
categorizations

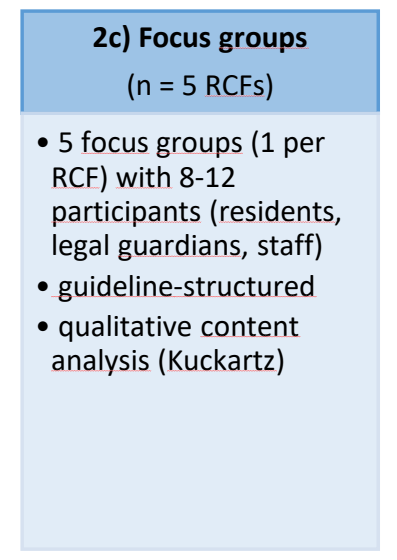




\section{1) Survey}

In order to generate quantitative data with regard to the use or avoidance of coercive measures, all RCFs in the study area will be surveyed.

\section{Sampling and recruitment}

All of the approximately $n=880$ RCFs for adults with disabilities in Bavaria will be included in the survey. The information required for contact and recruitment will be obtained by the Bavarian State Ministry for Health and Care. A response rate of 30\% ( $n=260$ completed questionnaires) is expected.

\section{Data collection}

An online questionnaire (45-120 minutes) will be administered via LimeSurvey. The survey link will be sent to the heads of the facilities by E-Mail with two telephone reminders after four and eight weeks. The questionnaire contains items describing the facilities, the use and avoidance of coercive measures/restraints, the justification for their use, and the alternatives to coercion used in the facilities. The survey will be completely anonymous. Pilot interviews will be conducted with $n=5$ RCFs.

Data analysis

First, the data will be analysed descriptively. Subsequently, statistical associations between variables (e.g., the frequency of use of coercive measures and characteristics of the facilities) will be evaluated using inferential statistics such as correlation analysis.

\section{2a) Observations}

Partially standardized, participatory, but passive observations will be conducted in five selected RCFs. This will allow us to examine stakeholder behaviours as well as the structural framework, conditions, and contexts to which they are associated [23]. In this study, the primary aim of this procedure is to allow for systematic and comprehensive identification of coercive measures and their contextual factors (e.g., structural and architectural conditions or concrete situations before, during, and after 
the use of coercive measures), even if these are not perceived as such by the individuals studied. This portion of the investigation will be conducted and reported according to the SRQR and STROBE Statement $[24,25]$.

\section{Sampling and recruitment}

The RCFs in which the observations will take place will be recruited via a contact form included in the survey (study part 1). The heads of facilities can use the form to express their interest in participating in the study part 2 (2a-2c). Five of these RCFs will then be selected using purposeful sampling [26]. Inclusion criteria for these RCFs are: being an RCF for adults with disabilities, formal registration in the study area, and signing a letter of intent. The sample will include RCFs of varying size (number of residents), distributed across different administrative districts, operated by different providers, located in both urban and rural areas, and providing different types of services. With regards to the target population, two of the RCFs will house primarily people with intellectual disabilities, two will house individuals with mental disabilities and substance use disorders, and one will have a diverse population. A total of at least $n=150$ residents should be indirectly included through the observations in the selected RCFs. All residents will be asked for voluntary consent to participate in the study. Residents who do not consent to study participation will not be part of the observations.

\section{Data collection}

This process will consist of direct, open, passively participating, third-party observations without stimulus. The observations will be partially structured and documented in the form of brief descriptions and assessments. Each RCF will be observed for a total of 24 hours, divided into three observation phases of eight hours each. The observation of each RCF will include periods in the daytime, nighttime, and weekend, and will be carried out by one or two members of the research team, depending on the size of the RCF. The observers will move freely in the RCF, and a member of the project staff will visit each location before the first observation phase. The findings obtained in this visit (e.g., architectural features of the RCF) will be documented in an inspection protocol. The 
staff and residents in the RCF will be aware of the presence and activities of the observers and informed about the aims of the study. The observation does not include interactions between observers and residents, but the observers will have the opportunity to talk to the staff of the facilities after observing situations in which restraints were used to inquire about reasons, possible alternatives, and experiences using/dealing with these alternatives if this information cannot be obtained by observation alone. Observers will record relevant information and impressions during the observation periods in observation logs. Following the observations, reports will be compiled based on the formulated observation protocols. The facility-based observation reports will serve as the primary data source for the analysis. A standardized questionnaire addressed to the heads of the facilities will be used to determine the structural features of the RCFs. The data will be combined anonymously after the observations have been completed.

\section{Data analysis}

The data for this stage will consist of the observation reports, inspection protocols, and responses to the written questionnaire administered to the heads of the facilities. The data for each RCF will be summarized in the course of the evaluation, and the resulting material will be evaluated by means of qualitative content analysis by extraction [27, 28]. In addition, the frequency of use of coercive measures and the data collected from the questionnaires will be analysed descriptively.

\section{2b) Document analysis}

In the RCFs selected for observation, relevant data will be collected from documents that relate to the organisations themselves and the residents indirectly included in the study. These data, which were non-reactive and produced independently of the research process, provide contextual information on the observed coercive measures, justifications, and use of chemical restraints.

\section{Sampling and recruitment}


Organizational documents and resident records will be collected from the five selected RCFs. The records of all residents (approx. $n=150$ ) in the five RCFs will be examined. Individual documents of residents that do not consent to study participation will not be included.

\section{Data collection}

Data collection for document analysis will take place in each of the five selected RCFs directly after the last observation phase. The collection will be carried out by members of the project staff who will have access to all relevant documents through the heads of the facilities and record the information on site. The facility-related documents included in the analysis will be as follows: agreements according to Social Security Code XII Section 75, guiding principles, house rules and rules of conduct, position plans, and duty rosters. Resident-related documents will include case files, support plans, medical records, and other documentation that may provide information on the use of coercive measures. Relevant information will be extracted from resident-related documents using a recording form, and facility-related documents (e.g., house rules) will be recorded as a whole. The final data set will be anonymous.

\section{Data analysis}

A large part of the recorded data will be evaluated using deductive content categorization. Some data (e.g., medication plans) will be evaluated using descriptive frequency counts.

\section{2c) Focus groups}

Heterogeneous focus groups will be carried out to validate previous knowledge and explore the connection of other findings to the experiences and perspectives of participants. The focus group approach is a valid method for the participatory integration of relevant stakeholders [29] and has the advantage of providing knowledge on group-specific perceptions, interpretations, and opinions that can be gained in a thematically focused manner [30]. This part of the study will be conducted and reported according to the COREQ Statement [31]. 


\section{Sampling and recruitment}

Participants ( $n=40-60$, consisting of staff of the facilities, residents, and their legal guardians, in as equal proportions as possible) will be recruited from the five selected RCFs. Staff and the residents' legal guardians will be approached by the heads of the facilities. The initial contact with potentially participating residents will take place through the staff of the facilities. Potential participants will be referred to the project staff for further planning of the focus groups. The selection of participants will be carried out by using a purposeful sampling strategy [26]. Inclusion criteria for the participants will be: working in the RCF or being a resident or legal guardian of a resident, mastery of the German language, and capacity to give informed consent. The focus groups will include participants of varying ages, genders, and length of stay in the RCFs.

\section{Data collection}

A focus group will be conducted in each of the RCF studied to validate the findings by capturing the perspectives, perceptions, and interpretations of stakeholders. Preliminary results of study parts $2 a$ and $2 \mathrm{~b}$ will be presented to the participants. The focus groups will take place within each of the RCF and be conducted by two members of the project staff, who will be responsible for moderating the discussion and logging information. Each focus group should include 8-12 participants and last 120180 minutes (including breaks). The sessions will be audiotaped if all participants agree. The focus groups will be conducted using standardized discussion guidelines to improve comparability and a short socio-demographic questionnaire will be used to describe the sample. The final data will be anonymous.

\section{Data analysis}

The discussions will be transcribed and evaluated using content-structuring content analysis [32]. Discussion protocols from the project staff and any results and process visualizations (e.g., flip charts) will be integrated into the analysis.

\section{Data synthesis and triangulation}


Due to the multi-phase design of the study, the data and results will be synthesized after each study phase is finalized. These results will then be used in the analysis of the subsequent study phase. At the end of the project, data triangulation will be conducted for the overall project by connecting the different perspectives and types of data.

\section{Quality assurance}

Data collection will be as standardized as possible (e.g. by using measuring instruments).

Participating observations will be carried out by members of the project staff with relevant professional training and experience in the fields of pedagogy and nursing. Participants will be trained in advance to counteract possible bias. Document analysis will be performed by project staff with medical-psychiatric knowledge. Ongoing self-reflection will be conducted and logged during both data collection and data analysis in all study phases. Interim results will be presented and discussed in the project team. In addition, expert workshops will be held in each of the seven administrative districts of Bavaria with stakeholder representatives, members of self-help groups, members of specialized agencies for RCF's and facility-staff in order to generate practical views on selected topics. Moreover, partial results will be continuously reported to a project advisory board consisting of government representatives, practitioners, welfare associations, stakeholder representatives, and (private) associations as well as experts from different research fields.

\section{Discussion}

Little is known about the use, causes, and frameworks surrounding the use of coercive measures in inpatient RCFs for adults with disabilities. Given this lack of data, it is difficult to make any statements about the occurrence and effectiveness of avoidance and alternative strategies to these encroachments on fundamental rights. In addition, inpatient RCFs for adults with disabilities are heterogeneous settings with people living with different diseases. The mixed-method design of this study will address the previous lack of research data. The results of study part 1 (survey) will provide an overview of the structural conditions and use of coercive measures in RCFs. Study part 2 
(participating observations, document analysis, focus groups) will provide insight into selected RCFs and allow for comparisons between actual practice and the self-reported data generated through the survey. These results will therefore be highly beneficial to further research in the field and could be used to provide recommendations for action that may improve current practice and raise further topics for research and practice in the field.

The participation of stakeholders from practice settings in several parts of the study increases the practical knowledge gained by this study. This information can be used to raise awareness of the topic and present successful strategies and concepts to address the use of coercive measures or their alternatives, thus indirectly reducing the use of coercive measures.

The strengths of the study lie in its multi-perspective design in a combination of forensic, medical and social sciences. The synthesis of different forms of data collection will allow for the generation of comprehensive findings. The project team is composed of researchers from several disciplines (therapeutic pedagogy, psychiatry, law, medical ethics, and social sciences), ensuring the findings will be interpreted from the perspectives of different areas of expertise.

The conditions of the COVID-19 pandemic will be considered in the participating observations and focus groups by implementing adequate hygiene practices.

\section{List of abbreviations}

RCFs - residential care facilities

\section{Declarations}

\section{Ethics approval and consent to participate}

Potential participants will be sent an invitation letter, an information sheet, and a consent form. The documents mentioned will also be made available to residents in plain language and audio form. The informed consent obtained from study participants is written. If participants (residents) are not able 
to give consent, their legal guardians can consent to their participation. This study was approved by the Ethics Board of the Ludwig-Maximilians-University of Munich (No. 20-1104).

\section{Consent for publication}

All authors have approved the manuscript for publication.

\section{Availability of data and materials}

Not applicable.

\section{Competing interests}

The authors declare that they have no competing interests.

\section{Funding}

This work is funded by the Bavarian State Ministry for Health and Care. The funding body supports the study financially. The funding body has no active part in generating and interpreting the results. Representatives of the funding body are members of the project advisory board.

\section{Authors' contributions}

MK and TH prepared the first manuscript version. PB, TSS, and AM added essential contributions. All authors read and approved the final manuscript.

\section{Acknowledgements}

Not applicable.

\section{References}

1. Bundesverfassungsgericht [Federal Constitutional Court]. Beschluss des Zweiten Senats vom 23.03.2011, Az. - 2 BvR 882/09. 2011. http://www.bverfg.de/e/rs20110323_2bvr088209.html. Accessed 17 April 2021. 
2. Henking T. Patientenrechte in der Psychiatrie im Kontext von Zwang. Recht \& Psychiatrie. 2016;34:155-63.

3. Bundesverfassungsgericht [Federal Constitutional Court]. Beschluss des Zweiten Senats vom 24.07.2018, Az. - 2 BvR 309/15. 2018.

http://www.bverfg.de/e/rs20180724_2bvr030915.html. Accessed 17 April 2021.

4. Flammer E, Frank U, Steinert T. Freedom Restrictive Coercive Measures in Forensic Psychiatry. Front Psychiatry. 2020;11:146.

5. Steinert T, Hirsch S, Goebel R, Snellgrove B, Flammer E. Reduction of coercive measures under routine conditions in psychiatric hospitals 2004-2019: Strong effects in old age psychiatry, much less in general psychiatry. Eur Psychiatry. 2020;63:e102.

6. Bayerisches Landesamt für Statistik [Bavarian State Office for Statistics]. Sozialhilfe in Bayern 2018. Teil 2: Empfängerinnen und Empfänger. München: Bayerisches Landesamt für Statistik; 2018.

7. Bayerisches Landesamt für Statistik [Bavarian State Office for Statistics]. Einrichtungen und betreute Wohnformen für volljährige Menschen mit Behinderung in Bayern 2018. München: Bayerisches Landesamt für Statistik; 2019.

8. Reinwald JR, Horten B, Dreßing H, Salize HJ. Structural Features and Use of Coercive Measures in Forensic Psychiatry throughout Germany. Psychiat Prax. 2021; doi:10.1055/a1391-0192.

9. Hirsch S, Steinert, T. Measures to Avoid Coercion in Psychiatry and Their Efficacy. Dtsch Arztebl Int. 2019;116:336-43.

10. Steinert T, Hirsch S. Implementierung der S3-Leitlinie Verhinderung von Zwang. Prävention und Therapie aggressiven Verhaltens bei Erwachsenen. Psychiatr Prax. 2019;46:294-6.

11. Thornicroft G, Farrelly S, Szmukler G, Birchwood M, Waheed W, Flach C, et al. Clinical outcomes of Joint Crisis Plans to reduce compulsory treatment for people with psychosis: a randomised controlled trial. Lancet. 2013;381:1634-41. 
12. Bowers L. Safewards: a new model of conflict and containment on psychiatric wards. J Psychiatr Ment Health Nurs. 2014;21:499-508.

13. Fährmann E, Hindrichs S. Praxisleitfaden: Bewegungs- und freiheitsbeschränkende Maßnahmen vermeiden. Das Recht auf Selbstbestimmung in Pflege und Betreuung. 1st ed. Regensburg: Walhalla \& Praetoria; 2018.

14. Hindrichs S, Fährmann E. Alternativen zu freiheitsentziehenden Maßnahmen. Pflegefachliche und rechtliche Grundlagen zur Fixierungsvermeidung. Regensburg: Walhalla; 2016.

15. Bayerisches Staatsministerium für Gesundheit und Pflege [Bavarian State Ministry for Health and Care]. Eure Sorge fesselt mich". Alternativen zu freiheitsentziehenden Maßnahmen in der Pflege. München: Bayerisches Staatsministerium für Gesundheit und Pflege; 2015.

16. Bayerisches Staatsministerium für Gesundheit und Pflege [Bavarian State Ministry for Health and Care]. Verantwortungsvoller Umgang mit freiheitsentziehenden Maßnahmen in der Pflege. München: Bayerisches Staatsministerium für Gesundheit und Pflege; 2015.

17. König J, Schibrowski M. FEM - Freiheitseinschränkende Maßnahmen. Gesetzliche Grundlagen, Praxisbeispiele, Alternativen. Hannover: Schlütersche Verlagsgesellschaft; 2013.

18. Rosemann M, Gallinat J, Heinz A, Krüger U. Vermeidung von Zwangsmaßnahmen im psychiatrischen Hilfesystem (ZVP). 2020. https://www.baggpv.de/fileadmin/downloads/ZVP_Gesamtbericht_final.pdf. Accessed 19 April 2021.

19. Steinhardt I, Speck A, Freyberger H. Blackbox geschlossene Heime. Psychosoziale Umschau. 2013;1:6-8.

20. Jenderny S, Schreiter J, Steinhart I. Psychiatrische Wohnheime in Deutschland - Transparenz und Strukturen. Psychiatr Prax. 2020;47:260-6.

21. Heinrich J. Aggression und Streß. Entlastung und Entspannung durch Abbau massiver Aggressionsformen. 4th ed. Weinheim: Deutscher Studien-Verlag; 1998.

22. Szmukler G, Appelbaum PS. Treatment pressures, leverage, coercion, and compulsion in mental health care. J Ment Health. 2008;17:233-44. 
23. Baker L. Observation: A complex research method. Library Trends. 2006;55171-89.

24. O'Brien BC, Harris IB, Beckman TJ, Reed DA, Cook DA. Standards for reporting qualitative research: a synthesis of recommendations. Acad Med. 2014;89:1245-51.

25. von Elm E, Altman DG, Egger M, Pocock SJ, Gotzsche PC, Vandenbroucke JP. The Strengthening the Reporting of Observational Studies in Epidemiology (STROBE) statement: guidelines for reporting observational studies. Ann Intern Med. 2007;147:573-7.

26. Palinkas LA, Horwitz SM, Green CA, Wisdom JP, Duan N, Hoagwood K. Purposeful sampling for qualitative data collection and analysis in mixed method implementation research. Adm Policy Ment Health. 2015;42:533-44.

27. Gläser J, Laudel G. Life with and without coding: Two methods for early-stagedata analysis in qualitative research aiming at causal explanations. Forum Qual Soc Res. 2013;14:5.

28. Gläser J, Laudel G. Experteninterviews und qualitative Inhaltsanalyse. 3rd ed. Wiesbaden: Springer; 2009.

29. Schöpf AC, Ullrich A, Nagl M, Farin E. Group health education in inpatient rehabilitation: Patients' role perceptions. Health Education Journal. 2016;75:289-305.

30. Zwick MM, Schröter R. Konzeption und Durchführung von Fokusgruppen. In: Schulz M, Mack B, Renn O, editors. Fokusgruppen in der empirischen Sozialwissenschaft. Wiesbaden: Springer; 2012. p. 24-48.

31. Tong A, Sainsbury P, Craig J. Consolidated criteria for reporting qualitative research (COREQ): a 32-item checklist for interviews and focus groups. Int J Qual Health Care. 2007;19:349-357.

32. Kuckartz U. Qualitative Inhaltsanalyse. Methoden, Praxis, Computerunterstützung. 3rd ed. Weinheim: Beltz/Juventa; 2016. 